\title{
Neurokognitive Effekte als Marker für antidepressives Ansprechen
}

Fragestellung: Die vorliegende randomisierte kontrollierte Studie hatte zum einen die Zielsetzung, das Nebenwirkungsprofil der antidepressiven Ketamintherapie im Hinblick auf potenzielle neurokognitive Beeinträchtigungen zu untersuchen. Zum anderen sollte die Frage geklärt werden, ob der neurokognitive Status von Patienten mit therapieresistenter Depression (TRD) als Ansprechprädiktor einer Ketamintherapie bei TRD dient.

Hintergrund: Die Ketamintherapie, verabreicht als Infusion, erlangt zunehmend einen Stellenwert in der Behandlung von Patienten mit TRD. In mehreren Metaanalysen wurde eine Ansprechrate von etwa $50 \%$ bei TRD ermittelt. Allerdings wurde auch gezeigt, dass die antidepressiven Effekte in einem kurzen Zeitraum von wenigen Tagen nachlassen, sodass die klinische Anwendung in Form repetitiver Therapiesitzungen erfolgen sollte. Das Nebenwirkungsprofil der Ketamintherapie bei TRD ist noch nicht abschließend geklärt. Zudem gibt es gegenwärtig keine Prädiktionsparameter bezüglich des Ansprechens auf eine Ketamintherapie bei TRD.

Murrough JW, Burdick KE, Levitch CF et al. Neurocognitive effects of ketamine and association with antidepressant response in individuals with treatment-resistant depression: a randomized controlled trial. Neuropsychopharmacology 2016; 40: 1084-90
Patienten und Methodik: Es erfolgte eine Randomisierung von 72 Patienten mit TRD in eine Ketamin- und eine Midazolamgruppe als Kontrollkollektiv. Die Gruppen zeigten keine Unterschiede im Hinblick auf Geschlecht, Krankheitsdauer und Anzahl der medikamentösen Therapieansätze. Zur Evaluation des neurokognitiven Status wurde die Measurement and Treatment Research Consensus Cognitive Battery (MCCB) vor dem Interventionszeitpunkt und sieben Tage danach angewendet. Zudem wurde vor der Intervention sowie 24, 48 und 72 Stunden danach die Montgomery-Åsberg-Depression-RatingSkala (MADRS) zur Verlaufskontrolle der depressiven Symptomatik herangezogen.

Ergebnisse: Die Verarbeitungsgeschwindigkeit als neurokognitive Domäne zeigte sich als valider Prädiktor eines Ansprechens auf Ketamin bei TRD. In der Midazolam- sowie in der Ketamingruppe wurde sieben Tage post interventionem eine signifikante Besserung der kognitiven Leistung im Vergleich zum präinterventionellen Status in den neurokognitiven Domänen Verarbeitungsgeschwindigkeit, sprachlicher Ausdruck und visuelles Lernen ermittelt.

Schlussfolgerungen: In dieser randomisierten klinischen Studie wurde gezeigt, dass eine Ketamintherapie bei TRD nach einmaliger Gabe zu keiner Beeinträchtigung neurokognitiver Funktionen führt. Die verbesserte Leistung in den drei Teilbereichen Verarbeitungsgeschwindigkeit, sprachlicher Ausdruck und visuelles Lernen, wurde auch in der Midazolamgruppe nachgewiesen und wird als unspezifischer Lerneffekt im Rahmen der zweifach durchgeführten MCCB-Testung interpretiert. Eine herabgesetzte Verarbeitungsgeschwindigkeit bei Patienten mit TRD geht mit einer gesteigerten Ansprechrate der Ketamintherapie einher.

\section{Ein weiterer Schritt in Richtung klinischer Routineanwendung}

Die vorliegende randomisierte kontrollierte Studie kann zukünftig die Nutzen-Risiko-Abwägung in der Entscheidung bezüglich einer Ketamintherapie bei therapieresistenter Depression (zum gegenwärtigen Zeitpunkt „off label“) beeinflussen. Denn es wurde mit der herabgesetzten Verarbeitungsgeschwindigkeit ein klinischer Prädiktionsmarker bezüglich des Ansprechens auf Ketamin ermittelt. Dies könnte die Nutzen-Seite durch eine bessere Auswahl von Patienten, die einer Ketamintherapie zugeführt werden, stärken. Die RisikoSeite dahingegen wird in Hinblick auf neurokognitive Funktionseinbußen als Nebenwirkung relativiert, da sich nach einer Ketamininfusion keine Einbußen der neurokognitiven Fähigkeiten ergaben.

Als Limitation ist zu nennen, dass der neurokognitive Status nach einer einmalig applizierten Ketamintherapie erhoben wurde, wohingegen in der klinischen Anwendung seriel- le Therapieeinheiten durchgeführt werden. Weiterhin ist als Einschränkung zu erwähnen, dass mit einem endgültigen Patientenkollektiv von $n=62$ ( $n=43$ in der Ketamin-, $n=19$ in der Midazolam-Gruppe) die Fallzahl recht niedrig ist.

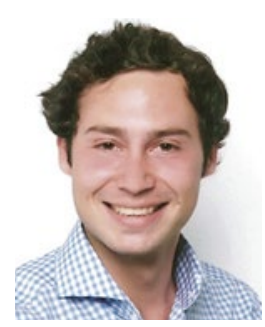

Johannes Weinbeer, Erlangen

Universitätsklinikum Erlangen, Psychiatrische und Psychotherapeutische Klinik E-Mail: johannes.weinbeer@uk-erlangen.de 